\title{
Identification of Chipless Sensors in Cluttered Environment From 3D Radar Imagery and Polarimetry
}

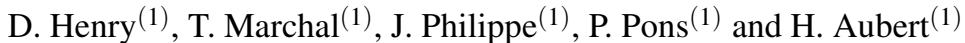

(1) LAAS-CNRS, 31400 Toulouse, FRANCE

\begin{abstract}
This paper reports a new method for the identification of chipless sensors based on 3D radar imagery and polarimetry. Chipless pressure sensors are wirelessly interrogated in a highly reflective environment with a $24 \mathrm{GHz}$ FM-CW radar. A novel algorithm of identification based on magnitude of radar echoes 3D distribution is proposed and applied to mitigate the clutter and identify multiple chipless pressure sensors. The electromagnetic footprint of sensors is defined and advantageously used for identification purposes.
\end{abstract}

\section{Introduction}

Identifying chipless sensors is a challenging problem that is particulary investigated in chipless RFID research fields. Innovative techniques of identification based on frequency shifts [1] up to complex design parameters, such as natural randomness of resonators [2] have been proposed. On the other hand, identification of tags can be performed by measuring the time delay of reflected impulses (see e.g. [3]). However, identifying chipless tags becomes very challenging when several contraints occur simultaneously, such as the presence of reflective environment and the requirement of a long range of interrogation (few meters and more). This task is even more challenging when tag are chipless sensors used to measure a physical quantity dedicated to a specific application. Very recently, the authors have been studied the feasibility to remotely interrogate chipless sensors in reflective environments by using millimeter-wave FM-CW (Frequency-Modulated Continuous-Wave) radars [4]. Radar echoes of the chipless sensors were detected from the 3D beamscanning of the scene, but this method requires to know the location of the sensors. Moreover, if several chipless sensors are interrogated, their identification was not addressed.

We propose in this paper a method for the identification of radar echoes of pressure chipless sensors interrogated in an industrial cluttered environment containing many reflective targets. The method is based on a combination of 3D radar images for different couplings of field polarization. In particular, radar echoes of the clutter are mitigated and radar echoes of two chipless pressure sensors are simultaneously identified from specific "electromagnetic footprints" thanks to so-called isolines of radar images.
The paper is organized as follows : the first section describes briefly the radar reader and the two pressure chipless sensors interrogated in an industrial hangar. The second section details the proposed algorithm of identification based on 3D radar imagery and polarimetry techniques. The third section describes how the identification can be performed by defining a specific electromagnetic footprint of pressure chipless sensors based on magnitude and 3D distribution of radar echoes.

\section{Reader and chipless sensors}

A frequency-modulated signal is transmitted by a FM$\mathrm{CW}$ radar of carrier frequency $f_{c}=23.8 \mathrm{GHz}$ and bandwidth $B=2 \mathrm{GHz}$. The transmitted power is $10 \mathrm{dBm}$. The front-end of the radar is composed of one transmitting $\left(T_{x}\right)$ channel and two receiving $\left(R_{x}\right)$ channels. The $T_{x}$ channel is connected to a narrow beamwidth lens antenna with an aperture angle of $6^{\circ}$ and gain of $28 \mathrm{dBi}$. The polarized electric field of the $T_{x}$ channel can be either vertically polarized (denoted here by the letter $V$ ) or horizontally polarized (denoted here by the letter $H$ ). The two receiving antennas $R_{x, 1}$ and $R_{x, 2}$ are identical horn antennas with gain of $20 \mathrm{dBi}$, and are respectively $V$ - and $H$-polarized.

We define the polarization configuration $p$ as the combination of transmitted and received electric fields in different polarizations. Two polarization configurations are used here for the measurements : (i) $p=V H$, when a $V$-polarized electric field is transmitted by the $T_{x}$ antenna and a $H$-polarized electric field is received by the $R_{x, 2}$ antenna; and (ii) $p=H V$ when a $H$-polarized electric field is transmitted by the $T_{x}$ antenna and a $V$-polarized electric field is received by the $R_{x, 1}$ antenna. Cross-polarization configurations are expected to offer higher SNR than copolarization configurations $(p=V V$ or $p=H H)$ and reduce the impact of the clutter [5]. The radar system is mounted on a mechanical plateform and performs a beamscanning in azimuth $(\varphi)$ and elevation $(\theta)$. During the beamscanning, chirps are transmitted periodically by the FM-CW radar with a repetition time of $T_{\text {rep }}=50 \mathrm{~ms}$.

To demonstrate the feasibility of the detection and identification of the chipless sensors, we perform a beamscanning in an industrial hangar where two chipless pressure sensors are placed. The beamscanned scene contains many objects, such as metallic pipes, grids and other reflective structures, as shown in Figure 1. The two chipless sensors 


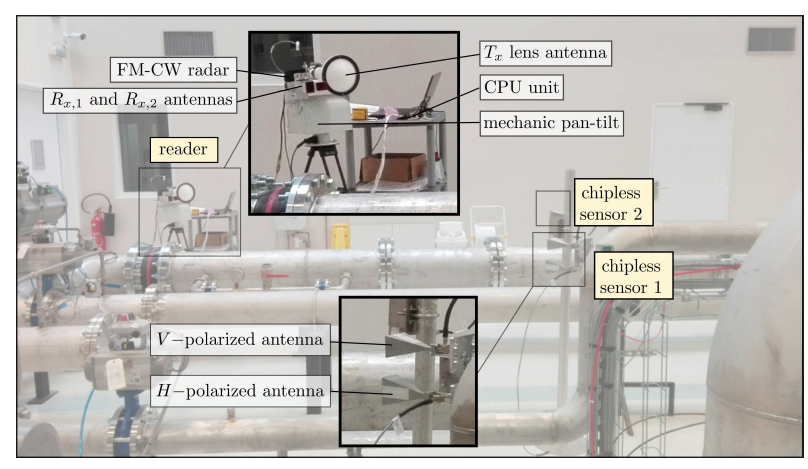

Figure 1. Beamscanned scene composed of many reflective objects. The reader system, composed of a FM-CW radar and a mechanic pan-tilt, is located at $2.5 \mathrm{~m}$ and $4.0 \mathrm{~m}$ from two chipless pressure sensors.

are located respectively at $2.5 \mathrm{~m}$ and $4.0 \mathrm{~m}$ from the radar. The chipless sensors are named respectively chipless sensor 1 and chipless sensor 2. The chipless pressure sensor is here a two-port network connected to two identical cross-polarized horn antennas with gain of $20 \mathrm{dBi}$. Between the antennas and the two-port network are connected delay lines of the same length in order to detect separatly the structural and sensing modes [4]. The radar echo magnitude of the sensing mode varies with the pressure applied on the two-port network. Details about the design of the chipless pressure sensor can be found in [6].

\section{Algorithm for identification of chipless sen- sors}

Radar echoes of the beamscanned scene are measured and analyzed from the algorithm described in Figure 2:

Step (i) - Raw data of measured radar echoes are registered during the mechanical beamscanning. Raw data are composed of $n$ demodulated time signals that are sampled into $N_{S}=1024$ time bins. Time bins are referred as fast time and the repetition time is referred as slow time. A single couple of angular values in azimuth and elevation $(\theta, \varphi)$ is associated to each demodulated signal. This first step, as well as steps (ii), (iii), (iv) and (v), are performed for $p=V H$ and $p=H V$;

Step (ii) - A Fast Fourier Transform (FFT) is applied on the raw data along the fast time axis in order to obtain $n$ beat frequency spectra of size $N_{S} / 2=512$ range bins. The size of one range bin is given by the depth resolution $d_{R}=c / 2 B=7.5 \mathrm{~cm}$, where $c=3.10^{8} \mathrm{~m} . \mathrm{s}^{-1}$ denotes the vacuum speed of light;

Step (iii) - We reconstruct the 3D radar image of the beamscanned scene in elevation/azimuth/range coordinates by associating a couple of angular values $(\theta, \varphi)$ to each range bin. The image is composed of voxels of size $d_{\theta} \times d_{\varphi} \times d_{R}$, where $d_{\theta}$ and $d_{\varphi}$ are the elevation and azimuth resolutions (here $d_{\theta}=d_{\varphi}=1^{\circ}$ );

Step (iv) - Radar echoes are segmented for each plane $(\theta, \varphi)_{k}$, where $k=1,2, \ldots, N_{S} / 2$, by using isolines. Isolines refer to lines along which the echo level is the same. The segmentation is based on the marching square algorithm and is detailed in [7]. The mean filter of size $5 \times 5$ pixels $^{2}$ is applied before the segmentation of each plane $(\theta, \varphi)_{k}$. The minimal threshold of echo level used for the segmentation is $-70 \mathrm{~dB}$. Surfaces of the segmented radar echoes are limited to the maximal threshold of 30 pixels $^{2}$;

Step (v) - Isolines for $p=V H$ and $p=H V$ are indexed and specific parameters are computed. $\mathscr{C}_{i}^{p}$ denotes the $i^{t h}$ isoline in polarization configuration $p, e_{\max , i}^{p}$ denotes the maximal value of echo level inside $\mathscr{C}_{i}^{p}$. Geometrical parameters are also registered, such as the surface of the isoline $\mathscr{C}_{i}^{p}$, denoted by $\mathscr{A}\left(\mathscr{C}_{i}^{p}\right)$;

Step (vi) - In order to identify radar echoes of the chipless sensors, couples of isolines for $p=V H$ and $p=H V$ that intersect in the same plane $(\theta, \varphi)_{k}$ are selected. This selection is applied for two reasons. First, the radar echoes of the sensing modes of two chipless sensors for $p=V H$ and $p=H V$ are located at the same range. And secondly, because the two cross-polarized horn antennas of the chipless sensors are close to each other (see Figure 1);

Step (vii) - The remaining couples of isolines are compared to known electromagnetic footprints of the chipless sensors. These footprints are defined with identifications parameters based on magnitude $\left(e_{V H-H V}\right.$, see Section 4, paragraph 1) and 3D distribution of radar echoes $\left(g_{\pi / 2}\right.$ and $g_{S}$, see Section 4, paragraph 2).

The algorithm is applied to the raw data measured from the beamscanned scene in polarization configurations $p=$ $V H$ and $p=H V$. Radar echoes are segmented with isolines (step (iv)) that are displayed on Figure 3(a) in elevation/azimuth/range coordinates for $p=V H$ (solid lines) and $p=H V$ (dashed lines). Due to the clutter, it seems difficult to identify radar echoes of the two chipless sensors : we have 28 radar echoes for $p=V H$ and 26 radar echoes for $p=H V$. To facilitate the detection, the step (vi) of the algorithm is applied. Remained isolines are displayed on Figure 3(b). We observed that 14 couples of isolines intersect for $p=V H$ and $p=H V$. It means that half of the radar echoes has been filtered by this process. However, we need a more selective method to improve the detection of the two chipless sensors. The step (vii) of the algorithm is applied on the remaining couple of isolines by using known electromagnetic footprints of chipless sensors 1 and 2 (see Section 4 for more details about the electromagnetic footprint). As displayed on Figure 3(c), only two couples of isolines remain. Couples of isolines in green and blue colors are identified as radar echoes of the chipless sensors 1 and 2 respectively. As it will be explained in Section 4, the efficiency of the identification depends on the characteristics of the electromagnetic footprint.

\section{Electromagnetic footprint of chipless pres- sure sensors}

A first parameter of identification based on the magnitude of radar echoes is proposed. For that purpose, we compute $e_{\text {max }}^{p}$ (defined in Section 3) which is an estimator of the applied pressure [5]. Values of $e_{\text {max }}^{V H}$ and $e_{\text {max }}^{H V}$ are displayed on 


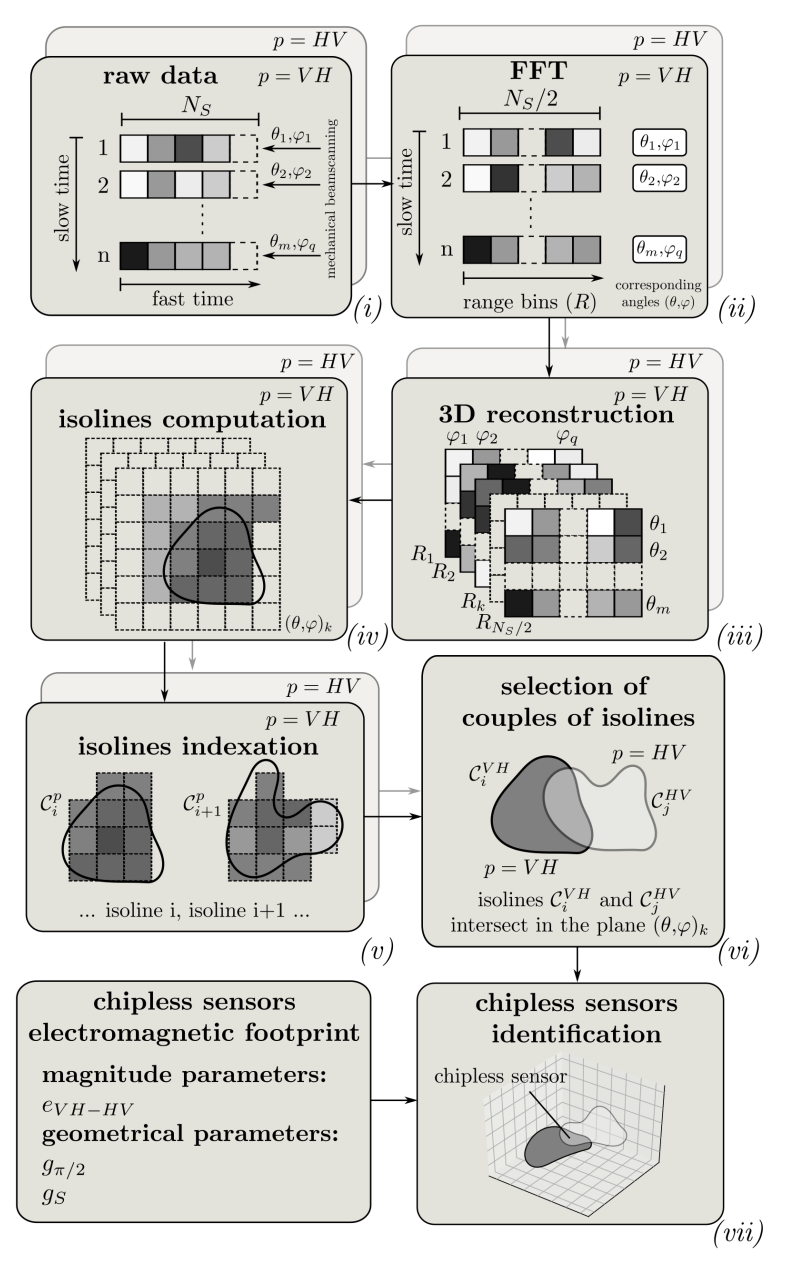

Figure 2. Algorithm for identification of chipless sensors decomposed into 7 steps. Radar echoes of chipless sensors are identified in step (vii) from electromagnetic footprints.

Figure 4(a) for $p=V H$ and $p=H V$ as a function of the applied pressure. We define here the following identification parameter based on the two polarization configurations as follows :

$$
e_{V H-H V}=e_{\max }^{V H}-e_{\max }^{H V}
$$

In the ideal case where the two-port network is symmetric, $e_{V H-H V}$ is constant and does not vary with the applied pressure. In practice, the two-port network is not symmetric and we have to deal with small variations of $e_{V H-H V}$, as displayed on Figure 4(b) for the chipless sensor 1 (in green) and the chipless sensor 2 (in blue). The term $\Delta_{e_{V H-H V}}$ in the figure denotes the range scale between minimal and maximal values of $e_{V H-H V}$. As a result, the identification between two chipless pressure sensors is possible if their respective range scales $\Delta_{e_{V H-H V}}$ have different values. We measure $\Delta_{e_{V H-H V}}$, sensor $1=-3 \pm 0.5 \mathrm{~dB}$ and $\Delta_{e_{V H-H V} \text {, sensor } 2}=-0.9 \pm 1 \mathrm{~dB}$. Then, we compute $e_{V H-H V}$ of the remaining 14 couples of isolines (see step (vi) of the algorithm). We note that :

(i) $e_{V H-H V}$ is found to be in the interval $\Delta_{e_{V H-H V}}$, sensor 1 for a unique couple of isolines : the chipless sensor 1 may be identified;

(ii) $e_{V H-H V}$ is found to be in the interval $\Delta_{e_{V H-H V}}$, sensor 2

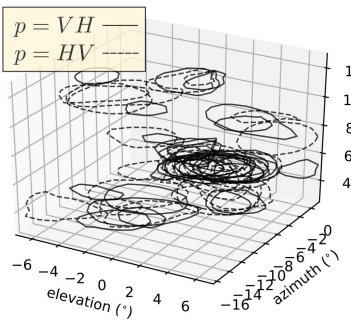

(a) (b)
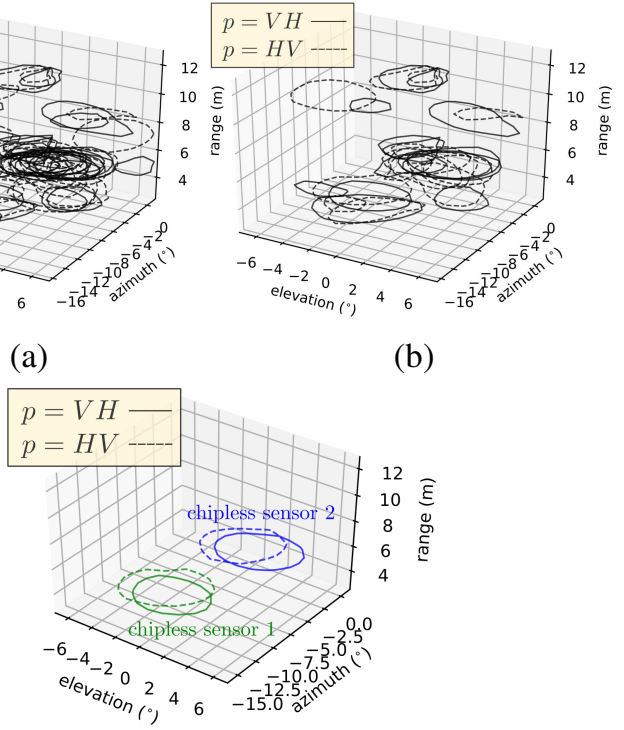

(c)

Figure 3. (a) Isolines of radar echoes from the reflective beamscanned scene for $p=V H$ (solid lines) and $p=H V$ (dashed lines) after the step (iv) of the algorithm. (b) After the step (vi) of the algorithm, half of the isolines has been removed. (c) When the step (vii) of the algorithm is applied, couples of isolines of the chipless sensors 1 (green color) and 2 (blue color) are easily identified.

for three couples of isolines. Two couples are associated with the clutter and the remaining couple with the chipless sensor 2. The chipless sensor 2 is not yet identified. Other identification parameters are needed to remove last radar echoes of the clutter.

We define in this paragraph two identification parameters based on the 3D distribution of radar echoes. The objective is to remove radar echoes of the clutter and identifiy the two chipless pressure sensors without ambiguity. The first geometrical identification parameter is defined as follows :

$$
g_{\pi / 2}^{p}=\frac{\mathscr{A}\left(\mathscr{C}^{p} \cap \mathscr{C}_{\pi / 2}^{p}\right)}{\mathscr{A}\left(\mathscr{C}^{p}\right)}
$$

As defined in Section $3, \mathscr{A}\left(\mathscr{C}^{p}\right)$ is the surface (in pixels ${ }^{2}$ ) of the isoline $\mathscr{C}^{p}$ for a polarization configuration $p \cdot \mathscr{C}_{\pi / 2}^{p}$ is identical to $\mathscr{C}^{p}$ but it is rotated by the angle of $p i / 2$ around its barycenter. $\mathscr{A}\left(\mathscr{C}^{p} \cap \mathscr{C}_{\pi / 2}^{p}\right)$ represents the surface (in pixels ${ }^{2}$ ) of the intersection between $\mathscr{C}^{p}$ and $\mathscr{C}_{\pi / 2}^{p}$. The identification parameter $g_{\pi / 2}^{p}$ is the indicator of $\pi / 2$ point reflection symmetry of the isoline used to segment a radar echo. If the isoline has a point reflection symmetry of $\pi / 2$, then $\mathscr{A}\left(\mathscr{C}^{p}\right)=\mathscr{A}\left(\mathscr{C}_{\pi / 2}^{p}\right)$ and $g_{\pi / 2}^{p}=1$. For a low symmetry, $g_{\pi / 2}^{p}$ tends toward zero. We define the identification parameter $g_{\pi / 2}=\frac{1}{2}\left(g_{\pi / 2}^{V H}+g_{\pi / 2}^{H V}\right)$. The second geometrical identification parameter is defined by:

$$
g_{S}=\frac{\mathscr{A}\left(\mathscr{C}^{V H}\right)}{\mathscr{A}\left(\mathscr{C}^{V H}\right)+\mathscr{A}\left(\mathscr{C}^{H V}\right)}
$$




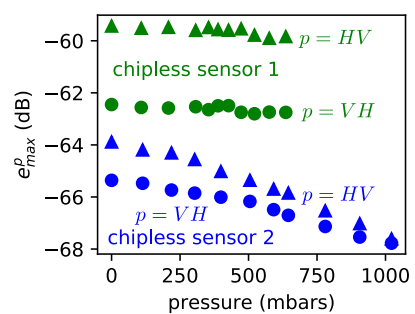

(a)

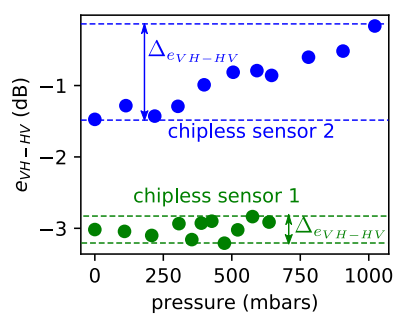

(b)
Figure 4. (a) Estimator $e_{\max }^{p}$ in function of the applied pressure of the chipless pressure sensors 1 (in green color) and 2 (in blue color) for $p=V H$ (circles) and $p=H V$ (triangles). (b) Identification parameter $e_{V H-H V}$ in function of the applied pressure of the chipless pressure sensors 1 (in green color) and 2 (in blue color).

This parameter is an indicator of surfaces difference between isolines $\mathscr{C}^{V H}$ and $\mathscr{C}^{H V}$. If isolines $\mathscr{C}^{V H}$ and $\mathscr{C}^{H V}$ have the same surface, $g_{S}=0.5$. If $\mathscr{A}\left(\mathscr{C}^{V H}\right)$ is much higher than $\mathscr{A}\left(\mathscr{C}^{H V}\right), g_{S}$ tends toward 1. If $\mathscr{A}\left(\mathscr{C}^{V H}\right)$ is much lower than $\mathscr{A}\left(\mathscr{C}^{H V}\right), g_{S}$ tends toward 0. Values of $g_{\pi / 2}$, $g_{S}$ and $e_{V H-H V}$ for each couple of isolines are displayed on Figure 5(a) for 11 different values of pressure applied on the chipless pressure sensors. Black crosses represent couples of isolines of the clutter. Green and blue boxes represent the electromagnetic footprint where the couples of isolines of chipless sensors 1 (green circles) and 2 (blue circles) are respectively located. The dimensions of the footprint (see the zoomed picture of the Figure 5(b)) are the range scale of each identification parameter : $\Delta_{e_{V H-H V}} \times \Delta_{g_{\pi / 2}} \times \Delta_{g_{S}}$. This electromagnetic footprint represents the minimal volume needed to detect the radar echoes of its corresponding chipless sensor in a given range of pressure. The number of dimensions of the electromagnetic footprint is the number of identification parameters (three in this example). The smaller the volume of the electromagnetic footprint, the easier the detection and identification of the chipless sensor. If a black cross (clutter) is inside the electromagnetic footprint, the identification of its corresponding chipless sensor might be altered. If two electromagnetic footprints intersect, the identification of their respective chipless sensors might not be possible for some values of pressure. We note that during our measurements, no black crosses are inside the boxes, and the two electromagnetic footprints do not intersect. It means that the three identification parameters $g_{\pi / 2}, g_{S}$ and $e_{V H-H V}$ are convenient to detect and identify the two chipless sensors in the industrial hangar.

\section{Conclusion}

A new algorithm for identification has been successfully applied on two interrogated pressure chipless sensors in a reflective environment. The clutter has been removed despite the presence of many reflective targets. Cross-polarization plays an important role in the success of the identification. Future works will study the limits of this method, such as the derivation of the maximal number of identifiable chipless sensors.

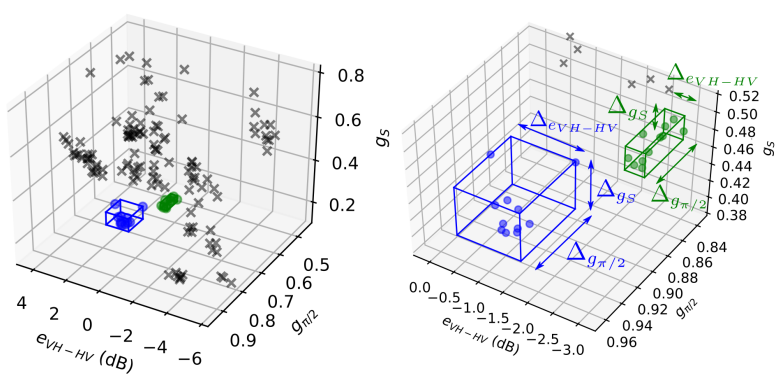

O chipless sensor $1 \quad$ chipless sensor $2 \quad \mathbf{X}$ clutter

(a)

(b)

Figure 5. (a) Values of identification parameters $g_{\pi / 2}, g_{S}$ and $e_{V H-H V}$ for each couple of isolines and for 11 values of pressure applied on the chipless pressure sensors. (b) Zoomed picture. Boxes of dimensions $\Delta_{e_{V H-H V}} \times \Delta_{g_{\pi / 2}} \times$ $\Delta_{g_{S}}$ are the electromagnetic footprints of respectively the chipless sensors 1 (green color) and 2 (blue color).

\section{References}

[1] O. Rance, E. Perret, R. Siragusa and P. LemaîtreAuger, "Effect of Distance for Chipless RFID Magnitude Coding," in IEEE Journal of Radio Frequency Identification, vol. 3, no. 2, pp. 77-82, June 2019.

[2] Z. Ali et al., "Detection of Natural Randomness by Chipless RFID Approach and Its Application to Authentication," in IEEE Transactions on Microwave Theory and Techniques, vol. 67, no. 9, pp. 3867-3881, Sept. 2019.

[3] M. M. Jatlaoui, F. Chebila, S. Bouaziz, P. Pons and H. Aubert, "Original identification technique of passive EM sensors using loaded transmission delay lines," The 40th European Microwave Conference, Paris, 2010, pp. 1106-1109.

[4] D. Henry, H. Aubert and P. Pons, "Radar Imaging Approach for Zero-Power Millimeter-Wave Wireless Sensors," 2019 IEEE International Conference on RFID Technology and Applications (RFID-TA), Pisa, Italy, 2019, pp. 89-94.

[5] D. Henry, T. Marchal, J.Philippe, P.Pons and H.Aubert, "Long-Range Zero-power Multi-sensing in Industrial Environment using Polarization Diversity and 3D Radar Imagery", IEEE International Microwave Symposium. Under review.

[6] J. Philippe, M. V. De Paolis, D. Henry, A. Rumeau, A. Coustou, P. Pons, and H. Aubert, "In-situ wireless pressure measurement using zero-power packaged microwave sensors," Sensors, vol. 19, no. 6, 2019.

[7] D. Henry and H. Aubert, "Isolines in 3D Radar Images for Remote Sensing Applications," 2019 16th European Radar Conference (EuRAD), PARIS, France, 2019, pp. 69-72. 